\title{
Partición de proteína de lactosuero en un sistema de extracción acuosa en dos fases
}

\author{
Whey protein partitioning in aqueous two-phase systems
}

\author{
Salvador Valle-Guadarrama', Ricardo Domínguez-Puerto ${ }^{2 *}$, Diana Guerra-Ramírez² \\ Departamento de Ingeniería Agroindustrial. Universidad Autónoma Chapingo. Km 38.5 Carretera México-Texcoco. C.P. \\ 56230, Texcoco, Estado de México. \\ 2 Laboratorio de Productos Naturales. Departamento de Preparatoria Agrícola. Universidad Autónoma Chapingo. Km 38.5 \\ Carretera México-Texcoco. C.P. 56230, Texcoco, Estado de México.
}

\section{RESUMEN}

El lactosuero es un subproducto de la fabricación de queso que contiene proteínas que pueden recuperarse, pero están muy diluidas y en mezcla con lactosa, lo que limita su aprovechamiento. La técnica de extracción acuosa en dos fases (ATPE, por sus siglas en inglés) es un procedimiento con potencial para separar proteínas. El objetivo fue evaluar el comportamiento de un sistema de extracción acuosa proteína- $\left(\mathrm{NH}_{4}\right)_{2} \mathrm{SO}_{4}$ aplicado para la recuperación de proteínas de lactosuero, atendiendo la reducción de lactosa. Se construyó un diagrama binodal de fases mediante una técnica de inspección de alto rendimiento. Se prepararon soluciones de $\left(\mathrm{NH}_{4}\right)_{2} \mathrm{SO}_{4}$ en concentraciones entre 22 y $40 \%$ usando lactosuero como disolvente. El volumen de la fase superior de los sistemas fue menor que el de la inferior, pero el incremento de $\left(\mathrm{NH}_{4}\right)_{2} \mathrm{SO}_{4}$ causó aumento gradual de la fase superior. La proteína de lactosuero se concentró en mayor cantidad en la fase superior alrededor del $80 \%$, en tanto que más del $79 \%$ de la lactosa se separó en la fase inferior. La técnica de ATPE tiene potencial para separar proteínas del lactosuero y la mejor alternativa se basa en sistemas con $34 \%$ de $\left(\mathrm{NH}_{4}\right)_{2} \mathrm{SO}_{4}$. Palabras clave: ATPE, inspección de alto rendimiento, sistemas proteína/ $\left(\mathrm{NH}_{4}\right)_{2} \mathrm{SO}_{4^{\prime}}$ proteínas de lactosuero, separación de lactosa.

\section{ABSTRACT}

Whey is a by-product of cheese manufacturing that has proteins that can be recovered, but they are very diluted and in a mixture with lactose, which limits their use. The aqueous two-phase extraction technique (ATPE) is a procedure with potential to separate proteins. The objective was to evaluate the behavior of an aqueous protein- $\left(\mathrm{NH}_{4}\right)_{2} \mathrm{SO}_{4}$ extraction system applied for the recovery of whey proteins, considering the reduction of lactose. A binodal phase diagram was constructed using a high throughput screening technique. Ammonium sulfate was dissolved at concentrations between 22 and $40 \%$ using whey as solvent. The volume of the top phase of the systems was less than that of the bottom one, but the increase in $\left(\mathrm{NH}_{4}\right)_{2} \mathrm{SO}_{4}$ caused a gradual increase at the top phase. Around $80 \%$ of whey protein concentrated at the top phase, while over $79 \%$ of lactose was at the bottom phase. The ATPE technique has the potential to separate proteins

\footnotetext{
*Autor para correspondencia: Ricardo Domínguez Puerto Correo electrónico: rdominguezp@chapingo.mx

from whey and the best alternative is based on systems with $34 \%\left(\mathrm{NH}_{4}\right)_{2} \mathrm{SO}_{4}$.

Key words: ATPE, high throughput screening, lactose separation, protein/ $\left(\mathrm{NH}_{4}\right)_{2} \mathrm{SO}_{4}$ systems, whey proteins.

\section{INTRODUCCIÓN}

El lactosuero es el residuo que permanece después de la recuperación de la cuajada enzimática o ácida en la producción de queso (Yadav et al., 2015). De $10 \mathrm{~kg}$ de leche, se producen generalmente de 8 a $9 \mathrm{~kg}$ de lactosuero, con composición (p/p) de 93.0-94.0\% de humedad, 4.5-6.0\% de lactosa, 0.6-1.1 \% de proteína, 0.8-1.0 \% de minerales, 0.05$0.90 \%$ de ácido láctico y 0.05-0.06 \% de grasas (Prazeres et al., 2012). Las proteínas de mayor presencia son a-lactoalbúmina (a-LA), $\beta$-lactoglobulina ( $\beta$-LG) y albúmina de suero bovino (BSA) con 60,30 y $6 \%$ de la proteína total, respectivamente (Capezio et al., 2005). La proteína de lactosuero es rica en aminoácidos ramificados y esenciales, péptidos funcionales, antioxidantes e inmunoglobulinas, los cuales pueden reducir el riesgo de contraer distintas asociadas a problemas cardiovasculares, hipertensión, obesidad, diabetes y cáncer (Patel, 2015). Debido a la importancia de estas proteínas es necesario aplicar alguna estrategia que permita aprovecharlas en la fortificación de alimentos, además de que con ello se reduce un problema de contaminación ambiental derivado del desecho del lactosuero.

La extracción acuosa en dos fases (ATPE, por sus siglas en inglés) es un procedimiento de separación líquidolíquido de diversos tipos de biomoléculas, donde se utilizan mezclas de compuestos que en ciertas concentraciones producen una solución verdadera en una sola fase, pero en otras generan la formación de dos fases inmiscibles, entre las cuales se reparten los compuestos a separar (Raja et al., 2011). El caso más común involucra el uso de un polímero como polietilenglicol, dextrano (Rosa et al., 2010), polivinil pirrolidona (Sadeghi y Maali, 2016), polióxido de etileno o polióxido de propileno (da Rocha et al., 2011) y una sal, como sulfato de amonio (Domínguez-Puerto et al., 2018), citrato de sodio (Rodríguez-Salazar y Valle Guadarrama, 2019) o fosfato de potasio (Alcântara et al., 2011). En la formación del sistema bifásico está involucrado un fenómeno de exclusión salina o salting-out, donde a medida que aumenta la concentración de sal se reduce la interacción del segundo componente con 
el agua, reduciendo su hidrofilicidad y dando lugar a una separación de dos fases inmiscibles (Gomes et al., 2017).

La técnica de ATPE ha sido probada con éxito para la separación de proteínas a partir de distintos materiales, tanto de origen vegetal (Balasubramaniam et al., 2003), como animal (Domínguez-Puerto et al., 2018; Kalaivani y Regupathi, 2015). Diversos trabajos se han enfocado a la separación de las proteínas del lactosuero mediante extracción acuosa en dos fases, con sistemas basados en polietilenglicol (PEG) y citrato de sodio (Perumalsamy y Murugesan, 2012), PEG y poliacrilato de sodio (Pereira Alcântara et al., 2014), PEG y citrato de sodio (Kalaivani y Regupathi, 2015), PEG y sulfato de amonio (Domínguez-Puerto et al., 2018), poli(etilenglicolran-propilenglicol)monobutiléter y fosfato de potasio (Jiang et al., 2017; Zhang et al., 2016), polivinil pirrolidona y fosfato de potasio (Mokhtarani et al., 2011) y PEG en mezcla con fosfato de potasio (Nandini y Rastogi, 2011), entre otros. Domínguez-Puerto et al. (2018) resaltaron que el lactosuero contiene también concentraciones relativamente altas de lactosa, lo cual debe ser atendido al recuperar las proteínas, pues muchas personas padecen de intolerancia a este disacárido (Jelen y Tossavainen, 2003). Domínguez-Puerto et al. (2018) también postularon que, por su estructura química, las proteínas pueden actuar por sí mismas como fase polimérica en un sistema de extracción acuosa en dos fases, para cumplir el objetivo de su separación del lactosuero. A este respecto, se ha reportado el uso de sistemas ATPE con proteínas en mezcla con polisacáridos, donde éstos actúan como agentes inductores del efecto de exclusión salina (Jara y Pilosof, 2011). Asimismo, se ha reportado que el incremento en el nivel de sacarosa en un sistema polisacárido-proteína ha favorecido la compatibilidad termodinámica de las mezclas y permitido la formación de una fase rica en proteína y, por ende, la separación de ésta (Sadeghi et al., 2021). Por otro lado, el sulfato de amonio $\left(\mathrm{AS},\left(\mathrm{NH}_{4}\right)_{2} \mathrm{SO}_{4}\right)$ es una sal comúnmente usada para la precipitación y estabilización de proteínas (Duong-Ly y Gabelli, 2014; Yang et al., 2010), que actúa, en forma similar a compuestos como los polisacáridos, por medio de un fenómeno de exclusión salina. Sin embargo, hasta hoy la partición de la proteína del lactosuero en un sistema proteína/sulfato de amonio no ha sido reportado, por lo cual el objetivo del trabajo fue evaluar el comportamiento de un sistema de extracción acuosa proteína- $\left(\mathrm{NH}_{4}\right)_{2} \mathrm{SO}_{4}$ aplicado para la recuperación de proteínas de lactosuero, atendiendo la reducción de lactosa

\section{MATERIALES Y MÉTODOS Lactosuero}

Se obtuvo lactosuero mediante filtrado de la cuajada resultante en un proceso de fabricación de queso panela a partir de leche entera de ganado Holstein, utilizando el método descrito por Lobato-Calleros et al. (2009). El suero se mantuvo en congelación a $-20^{\circ} \mathrm{C}$ y porciones de éste fueron separadas periódicamente para el desarrollo experimental. El lactosuero se evaluó con equipo Milko Scan FT1 (Foss, Denmark) y tuvo $0.715 \%( \pm 0.038)$ de grasa, $13.1( \pm 0.58) \mathrm{mg} \mathrm{mL}^{-1}$, de proteína y $49.5( \pm 7.56) \mathrm{mg} \mathrm{mL}^{-1}$ de azúcares.

\section{Diagrama binodal de fases}

Se construyó un diagrama binodal de fases utilizando sulfato de amonio $\left.\left(\mathrm{NH}_{4}\right)_{2} \mathrm{SO}_{4} ; \mathrm{AS}\right)$, agua destilada y lactosuero como fuente de proteína. Se utilizó la técnica de inspección de alto rendimiento (HTS; high throughout screening) (Bensch et al., 2007, Spyropoulos et al., 2010) con modificaciones. Se eligieron ocho intervalos de concentración de AS, en rangos de 14.0-20.0 (denominado R1), 15.0-21.0 (R2), 16.0-22.0 (R3), 18.0-24.0 (R4), 20.0-26.0 (R5), 22.0-28.0 (R6), 24.0-30.0 y 30.0$36.0 \%$ (R8). En cada intervalo la concentración de proteína se mantuvo constante con valores de $0.91,0.55,0.36,0.18$, $0.09,0.045,0.018$, y $9.68 \times 10^{-3} \%$, respectivamente (Figura 1). Cada sistema se dejó en reposo durante 24 h, tras lo cual se identificó la formación de un sistema en una o dos fases separadas. En cada intervalo (R1 a R8) se identificó la mezcla o sistema con menor concentración de AS que formó un sistema bifásico. Los estados identificados se rotularon con la notación $\mathrm{B} 1$ a B8, respectivamente, se usaron para construir una curva binodal de fases $y$, asimismo, para delimitar las regiones monofásica y bifásica. Las concentraciones de AS y proteína de los estados B1 a B8 se ajustaron a la Ecuación 1 (Merchuk et al., 1998) por medio de regresión no lineal usando el software Matlab (The Mathworks Inc., 1990-2008), donde $x_{A S}^{b i n}(\%)$ y $y_{p r o}^{b i n}(\%)$ son concentraciones de equilibrio de AS y proteína sobre la curva binodal y $k_{1}, k_{2}$ y $k_{3}$ son constantes de regresión. Todas las rutinas experimentales se realizaron a $25^{\circ} \mathrm{C}$.

$$
y_{\text {pro }}^{\text {bin }}=k_{1} e^{\left(k_{2}\left(x_{A S}^{b i n}\right)^{0.5}-k_{3}\left(x_{A S}^{b i n}\right)^{3}\right)}
$$

El diagrama de fases se completó con una línea de operación (TL) en la región bifásica a través de la preparación de disoluciones de sulfato de amonio (AS) en concentra-

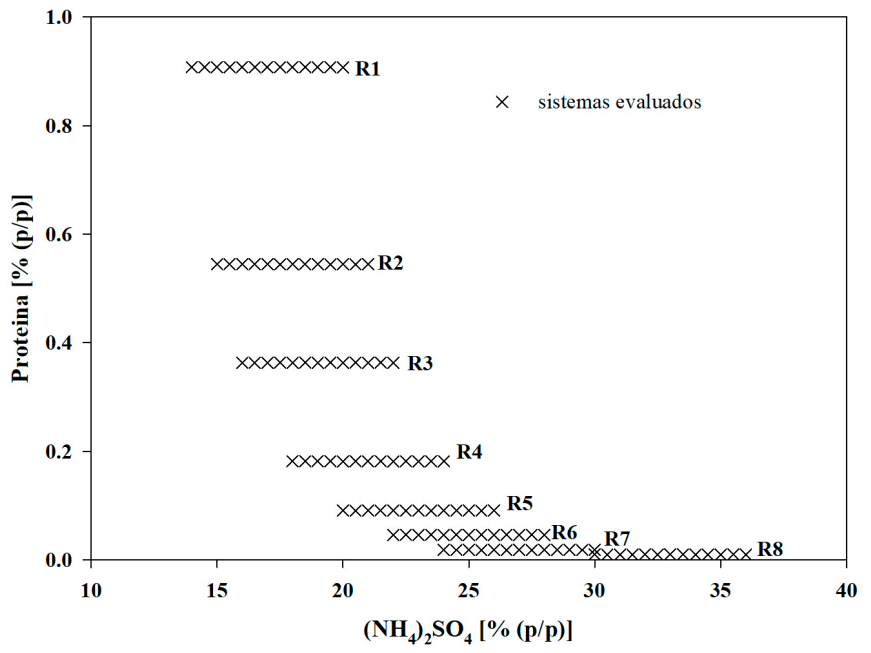

Figura 1. Sistemas evaluados para encontrar la línea binodal. Concentraciones AS/proteína (\%/\%): R1 = 14-20 /0.91; R2=15-21 /0.55; R3= 16-22 /0.36; $\mathrm{R} 4=18-24 / 0.18 ; \mathrm{R} 5=20-26 / 0.09 ; \mathrm{R} 6=14-20 / 0.045 ; \mathrm{R} 7=20-30 / 0.018 ; \mathrm{R} 8=$ $14-20 / 9.68 \times 10^{-3}$.

Figure 1. Evaluated systems to locate binodal curve. AS/protein concentrations (\%/\%): R1 = 14-20 /0.91; R2=15-21/0.55; R3=16-22 /0.36; R4= $18-24 / 0.18 ; \mathrm{R} 5=20-26 / 0.09 ; \mathrm{R} 6=14-20 / 0.045 ; \mathrm{R} 7=20-30 / 0.018 ; \mathrm{R} 8=14-20$ $/ 9.68 \times 10^{-3}$. 
ciones de $22,26,30,34,36,38$ y $40 \%$, usando lactosuero como disolvente. Estas mezclas se rotularon como P1 a P7, respectivamente. Con base en la concentración original del lactosuero se determinó la concentración de proteína en cada una de las mezclas. Los datos se ajustaron a la Ecuación (2) y el resultado se graficó sobre el diagrama de fases, donde $y_{p r o}^{T L}$ es concentración de proteína, $x_{A S}^{T L}$ es concentración de sulfato de amonio y $k_{4}$ y $k_{5}$ son constantes de regresión.

$$
y_{\text {pro }}^{T L}=k_{4}+k_{5} x_{A S}^{T L}
$$

\section{Parámetros de partición}

Los volúmenes de las fases superior $\left(V_{T}, \mathrm{~mL}\right)$ e inferior $\left(V_{B^{\prime}} \mathrm{mL}\right)$ se midieron con una probeta. Con ellos se determinó la relación de volúmenes $(R)$, como el cociente de $V_{T}$ entre $V_{B}$ (Ecuación 3), para las condiciones de operación localizadas sobre la línea TL.

$$
R=\frac{V_{T}}{V_{B}}
$$

Cada mezcla se mantuvo en reposo a $25^{\circ} \mathrm{C}$ durante 24 h para permitir la formación de sistemas bifásicos. Posteriormente, las fases se separaron y se evaluaron en términos de , concentración de proteína y concentración de lactosa. Para cada componente se determinó un coeficiente de partición $(K)$ mediante la Ecuación (4) y rendimientos de separación $\left(Y_{T^{\prime}}\right.$ $Y_{B}$ ) en las fases superior $(T)$ e inferior $(B)$ con las Ecuaciones (5) y (6), respectivamente, donde $C_{T}, c_{B}$ y $c_{0}$ son concentraciones ( $\mathrm{mg} \mathrm{mL}^{-1}$ ) de proteína o lactosa en $T$, en $B$ y en el lactosuero original, respectivamente. Asimismo, $V_{0}$ es el volumen $(\mathrm{mL})$ de lactosuero adicionado.

$$
\begin{aligned}
& K=\frac{c_{T}}{c_{B}} \\
& Y_{T}(\%)=\left(\frac{c_{T} V_{T}}{c_{0} V_{0}}\right) \times 100 \\
& Y_{B}(\%)=\left(\frac{c_{B} V_{B}}{c_{0} V_{0}}\right) \times 100
\end{aligned}
$$

\section{Evaluación de variables}

La concentración de proteína se determinó con el método de absorción a $220 \mathrm{~nm}$ (Kamizake et al., 2003), con un espectrofotómetro Hach DR 5000 UV-Vis (Hach, México). Las determinaciones se apoyaron con una curva estándar de albúmina de suero bovino (BSA) en un rango de 10 a $80 \mathrm{mg}$ $\mathrm{mL}^{-1}$ y los resultados se expresaron como $\mathrm{mg}$ equivalentes de BSA por $\mathrm{mL}$ (mg BSA $\mathrm{mL}^{-1}$ ). La concentración de lactosa se midió con el método de fenol-sulfúrico (DuBois et al., 1956), en un espectrofotómetro con lector de microplacas (Biotek, Sinergy 2, BioTeck Instruments, USA) y usando una curva estándar de lactosa en el rango de 10 a $200 \mathrm{mg} \mathrm{mL}^{-1}$.

\section{Análisis de datos}

Los datos de relación de volúmenes $(R)$, concentración de proteína y lactosa en las fases, rendimientos de separación $\left(Y_{T}, Y_{B}\right)$ y coeficiente de partición $(K)$ se sometieron a análisis de varianza y a pruebas de comparación de medias mediante el estadístico de Tukey con nivel de significancia de 0.05. Se usó el Programa SAS (SAS Institute Inc., 1999) como apoyo para el análisis de datos. Todas las evaluaciones se realizaron por triplicado.

\section{RESULTADOS Y DISCUSIÓN Diagrama binodal de fases}

El método de inspección de alto rendimiento (HTS) permitió verificar que, para valores constantes de contenido de proteína, el incremento de la concentración de $\left(\mathrm{NH}_{4}\right)_{2} \mathrm{SO}_{4}$ causó la transición de sistemas monofásicos a sistemas donde se observó la separación en dos fases. El suero tuvo contenido de proteína de $1.21( \pm 0.05) \%$, con lo cual se calculó el contenido proteico de todas las mezclas evaluadas. Los puntos binodales identificados tuvieron concentración de $\left(\mathrm{NH}_{4}\right)_{2} \mathrm{SO}_{4}$ /proteína (\%/\%) de 18.25/0.9075 (B1), 19.50/0.5445 (B2), 21.00/0.3630 (B3), 22.50/0.1815 (B4), 23.50/0.0908 (B5), 24.50/0.0460 (B6), 26.00/0.0182 (B7) y, 34.00/9.68×10-3 (B8) (Figura 1). Con estos datos se ajustó la curva binodal (Ecuación 2) con valores de $18.98,-0.3095,2.852 \times 10^{-4}$ en las constantes $k_{1}, k_{2}$ y $k_{3}$, respectivamente y coeficiente de determinación $\left(r^{2}\right)$ igual a 0.9903 (Figura 2). Por otro lado, el límite de solubilidad del sulfato de amonio es $43.4 \%$ a $25^{\circ} \mathrm{C}$ (Wingfield, 2016). En tal sentido, la curva binodal se extrapoló, mediante la Ecuación (2), hasta la concentración de sulfato de amonio de 40 $\%$, de donde se identificó el estado B9 con concentración de proteína de $8.80 \times 10^{-3} \%$ (Figura 2).

El diagrama binodal de fases se completó con la construcción de una línea de operación en la región bifásica. Las condiciones usadas tuvieron concentración AS/proteína (\%/\%) de 22/0.94 (P1), 26/0.90 (P2), 30/0.85 (P3), 34/0.80 (P4),

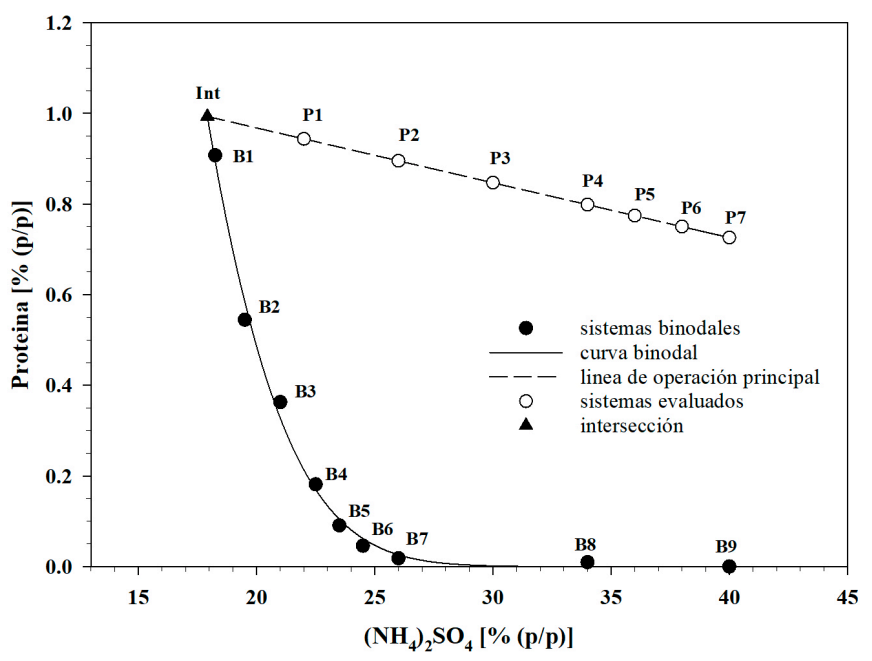

Figura 2. Diagrama de fases con curva binodal y sistemas experimentales evaluados.

Figure 2. Phases diagram with binodal curve and evaluated experimental systems. 
36/0.77 (P5), 38/0.75 (P6) y 40/0.73 \% (P7) (Figura 2). El ajuste de estos datos a la Ecuación 2 tuvo constantes de regresión $k_{4}$ y $k_{5}$ de $1.2083 \%$ y 0.0120 , respectivamente, y coeficiente de determinación $\left(r^{2}\right)$ igual a 0.9979. Las Ecuaciones 1 y 2 se resolvieron simultáneamente y se determinó la intersección (Int, Figura 2) entre ellas en la condición con $17.9262 \%$ de sulfato de amonio y $0.9932 \%$ de proteína. En la Figura 3 se compara la línea binodal obtenida con PEG4000 y sulfato de amonio por Domínguez-Puerto et al. (2018) con la línea binodal para el sistema proteína- $\left(\mathrm{NH}_{4}\right)_{2} \mathrm{SO}_{4}$ obtenida en este trabajo, las cuales se diferenciaron en el hecho de que en la primera se utilizó agua como disolvente (línea sólida), en tanto que para la segunda se usó lactosuero más agua (línea punteada). La curva binodal obtenida con proteína tuvo rango de concentración de 17.92 a $40.00 \%$ de $\left(\mathrm{NH}_{4}\right)_{2} \mathrm{SO}_{4}$, mientras que la obtenida con PEG4000, el rango fue de 3.46 a $34.71 \%$. En la Figura 3 se muestran las condiciones de operación evaluadas en el presente trabajo (P1 a P7), y condiciones identificadas por los puntos 1 a 7 , las cuales fueron evaluadas para el sistema PEG4000/AS por (Domínguez-Puerto et al., 2018), quienes encontraron que a medida que se redujo la concentración de PEG en el sistema se incrementó la concentración de proteína en la fase superior y recomendaron el uso de sistemas sin PEG y concentración de $\left(\mathrm{NH}_{4}\right)_{2} \mathrm{SO}_{4}$, de $34.0 \%$ para conseguir rendimientos de separación de proteína mayores a $92 \%$. A este respecto, los datos del presente trabajo mostraron que la recomendación de Domínguez-Puerto et al. (2018) coincidió con la intersección de las líneas de operación evaluadas en ambos trabajos (Figura 3). Asimismo, el hecho de que la curva de operación evaluada en el presente trabajo se ubicó en la región bifásica, confirmó que la separación de proteínas de lactosuero puede realizarse sin el uso de un polímero como polietilenglicol y que las proteínas, por su estructura química, pueden por sí mismas fungir como componente polimérico en su separación.

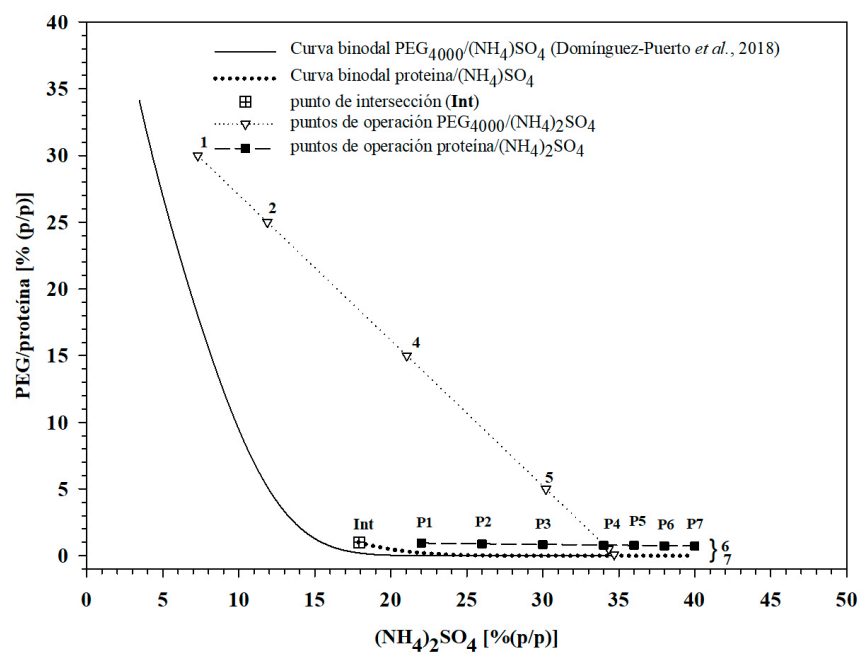

Figura 3. Comparación de curvas binodales entre los sistemas $\left(\mathrm{NH}_{4}\right)_{2} \mathrm{SO}_{4} /$ $\mathrm{PEG}_{4000}(1,2,4,5,6$ y 7$)$ y proteína/ $\left(\mathrm{NH}_{4}\right)_{2} \mathrm{SO}_{4}(\mathrm{P} 1$ a P7) y sus respectivos puntos de operación.

Figure 3. Binodal curves comparison between $\left(\mathrm{NH}_{4}\right)_{2} \mathrm{SO}_{4} / \mathrm{PEG}_{4000}(1,2,4$, $5,6$ y 7$)$ and protein/( $\left(\mathrm{NH}_{4}\right)_{2} \mathrm{SO}_{4}(\mathrm{P} 1$ a P7) systems and their corresponding operation points.
Por otro lado, la curva binodal del sistema proteína/ $\left(\mathrm{NH}_{4}\right)_{2} \mathrm{SO}_{4}$, resultó desplazada hacia la derecha con relación a la curva binodal construida con PEG4000/( $\left(\mathrm{NH}_{4}\right)_{2} \mathrm{SO}_{4}$. Este fenómeno pudo ocurrir debido a que la proteína tiene menor solubilidad en agua en comparación con el polietilenglicol, el cual tiene un grupo funcional éter (metoxi) de manera periódica en su estructura y puede ligar de dos a tres moléculas de agua por unidad (Turecek et al., 2016), lo que lo hace altamente soluble. Sin embargo, aunque las proteínas del lactosuero son solubles, la interacción con el agua es menor y poseen una estructura mayor con pesos moleculares del orden de 14, 18 y $66 \mathrm{kDa}$ para a-LA, $\beta$-LG y BSA, respectivamente (Madureira et al., 2007), que contrasta con el caso del polietilenglicol, cuyo valor es de 4 kDa para PEG4000.

\section{Relación de separación de volúmenes}

La relación entre los volúmenes separados $(R)$ varió en forma sigmoidal (Figura 4) y los datos se ajustaron bien $\left(r^{2}=0.9802\right)$ a la Ecuación 7 , donde $k_{6}, k 7, k_{8}$ y $k_{9}$ son constantes de regresión que tuvieron valores de 0.0619, 32.7401, 2.4499 y 0.2953 , respectivamente y es concentración de sulfato de amonio.

$$
R=k_{6}+k_{7} e^{-e^{-\left(\frac{x_{A S}-k_{8}}{k_{9}}\right)}}
$$

El valor de se mantuvo constante en los sistemas P1 a $\mathrm{P} 3$, con valor aproximado de 0.06 , sin diferencia significativa entre ellos y con un volumen en la fase inferior que fue 16 veces mayor que en la fase superior. Entre los estados P3 y P6 el valor de $R$ aumentó significativamente hasta valores de 0.30 y 0.35 en las condiciones P6 y P7, sin diferencia significativa entre estas dos últimas (Figura 4), lo que sugirió que el efecto de la composición sobre la relación de volúmenes dejó de ser significativo a partir de una concentración de sulfato de amonio de $38 \%$, lo cual se explica por el acercamiento a la

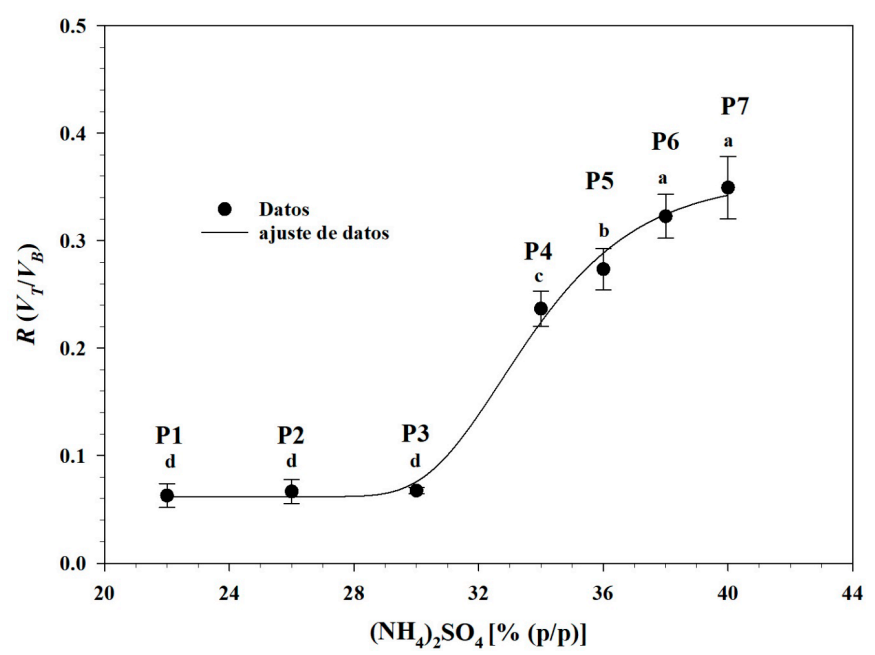

Figura 4. Volúmenes de separación de los sistemas experimentales proteí$\mathrm{na} / \mathrm{sulfato}$ de amonio. Letras diferentes indican diferencias significativas, de acuerdo a la prueba de Tukey $(\mathrm{p}<0.05)$.

Figure 4. Volume ratio of experimental operation points of the protein/ammonium sulphate systems. Different letters indicate significant differences, according to Tukey's test $(p<0.05)$. 
condición de saturación del sulfato de amonio, con valor de $43.4 \%$ (Wingfield, 2016). Por otro lado, la relación de volúmenes normalmente disminuye a medida que disminuye la presencia del componente polimérico y aumenta el componente salino en el sistema ATPE (Raja et al., 2011), lo cual contrastó con el comportamiento observado en el presente trabajo. La formación del sistema bifásico está determinada por un fenómeno de exclusión salina o salting-out (Gomes et al., 2017). En el presente trabajo, a medida que aumentó la concentración de $\left(\mathrm{NH}_{4}\right)_{2} \mathrm{SO}_{4}$ se incrementó la interacción iondipolo entre esta sal y el agua, con lo cual se incrementó el carácter hidrofóbico de las proteínas (Murillo-Martínez et al., 2013) y con ello su exclusión (efecto salting-out), dando lugar a una fase separada proteínica. Sin embargo, el aumento de la relación de volúmenes $(R)$ sugirió que otros componentes quedaron también alojados en esa fase y causaron flujo de agua hacia la fase superior.

\section{Concentración de proteína}

La concentración de proteína osciló de 48.63 a 30.08 $\mathrm{mg} \mathrm{mL}^{-1}$ y de 6.56 a $2.02 \mathrm{mg} \mathrm{mL}^{-1}$ en las fases superiores $\mathrm{e}$ inferiores de los sistemas, respectivamente, durante la transición de la condición P1 a la P7 (Figura 5). La diferencia entre ambas fases fue significativa $(p \leq 0.05)$, lo que confirmó que la proteína tendió a migrar hacia la fase superior y que ésta se formó por el efecto de exclusión salina causado por el $\left(\mathrm{NH}_{4}\right)_{2} \mathrm{SO}_{4}$. Este hecho fue más evidente en la transición entre los estados P1 y P3, donde la concentración de proteína se redujo en la fase inferior y aumentó en la superior y donde la relación de volúmenes no cambió, es decir, que las dimensiones volumétricas de la fase superior en relación con la inferior no se modificaron (Figura 4). Aunque el volumen de la fase inferior disminuyó en relación con el de la fase superior entre los estados P3 y P7, la concentración de proteína continúo disminuyendo en la fase inferior a medida que aumentó el

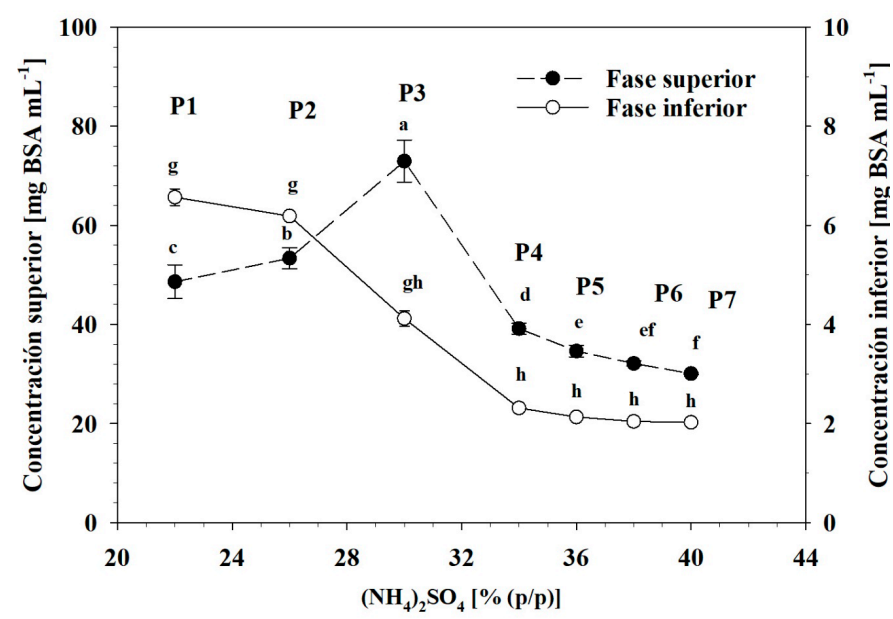

Figura 5. Concentración de proteína en la fase superior e inferior de los sistemas evaluados. Letras diferentes indican diferencias significativas, de acuerdo a la prueba de Tukey $(p<0.05)$.

Figure 5. Top and bottom phase protein concentration of evaluated operation points. Different letters indicate significant differences, according to Tukey's test $(p<0.05)$.
$\left(\mathrm{NH}_{4}\right)_{2} \mathrm{SO}_{4}$. Sin embargo, en la fase superior la concentración de proteína disminuyó también en el mismo rango de variación del sulfato de amonio, pero el comportamiento fue más bien causado por un efecto de dilución, pues el volumen de esa fase aumentó a medida que aumentó la presencia de $\left(\mathrm{NH}_{4}\right)_{2} \mathrm{SO}_{4}$. Con base en estos datos, el coeficiente de partición de proteína entre las fases $\left(K_{\text {pro }}\right)$ aumentó de 7.42 a 17.74 en la transición de P1 a P3 (Figura 6), aunque en la región de P3 a P7 este valor se redujo a 14.86, derivado del efecto de dilución de la fase superior.

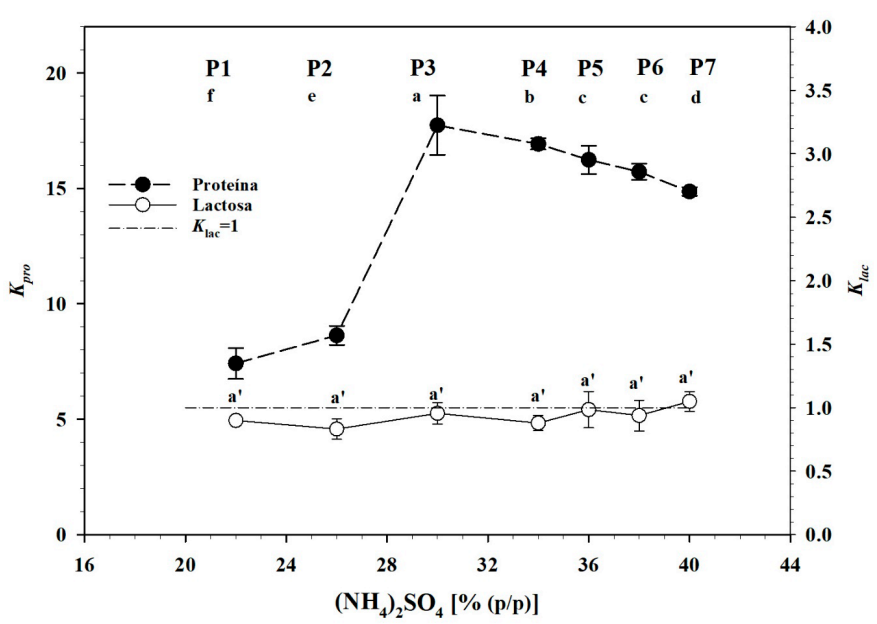

Figura 6. Coeficiente de partición para los sistemas evaluados. Letras diferentes indican diferencias significativas, de acuerdo a la prueba de Tukey $(p<0.05)$.

Figure 6. Partitioning coefficient for evaluated operation points. Different letters indicate significant differences, according to Tukey's test $(p<0.05)$.

\section{Concentración de lactosa}

La concentración de lactosa osciló entre 36.72 y 40.62 $\mathrm{mg} / \mathrm{mL}$ en las fases superiores y entre 38.06 y $47.06 \mathrm{mg} / \mathrm{mL}$ en las fases inferiores de los sistemas evaluados sobre la línea de operación (Figura 7). La diferencia de concentración de lactosa entre fases no fue significativa en las condiciones P3 a P7 y, aunque sí lo fue en los casos P1 y P2, en realidad no tuvo importancia práctica. La relación de la concentración de un compuesto en la fase superior y la concentración de éste en la fase inferior está determinada por el coeficiente de partición ( $K$; Ecuación 4), donde un valor mayor a 1 indica mayor concentración del compuesto en la fase superior, en tanto que un valor menor a la unidad indica mayor concentración en la fase inferior. El coeficiente de partición de lactosa $\left(\mathrm{K}_{\text {lac }}\right)$ fue $0.902( \pm 0.01)$ en $\mathrm{P} 1,0.836( \pm 0.030)$ en $\mathrm{P} 2$, $0.944( \pm 0.029)$ en P3, $0.880( \pm 0.023)$ en P4, $1.005( \pm 0.049)$ en P5, $0.952( \pm 0.043)$ en P6 y $1.040( \pm 0.027)$ en P7. Estos valores indicaron que la lactosa se obtuvo con concentración sólo ligeramente mayor en la fase inferior que en la fase superior de los sistemas y que no hubo una tendencia a que este disacárido se concentrará de forma clara en alguna de las fases, sino que quedó aparentemente repartido entre ambas, con concentración aproximadamente similar. Domínguez-Puerto et al. (2018) reportaron valores promedio de $K_{\text {tac }}$ de 0.58 en sistemas basados en polietilenglicol 4000 y sulfato de amo-

Volumen XXIII, Número 3 


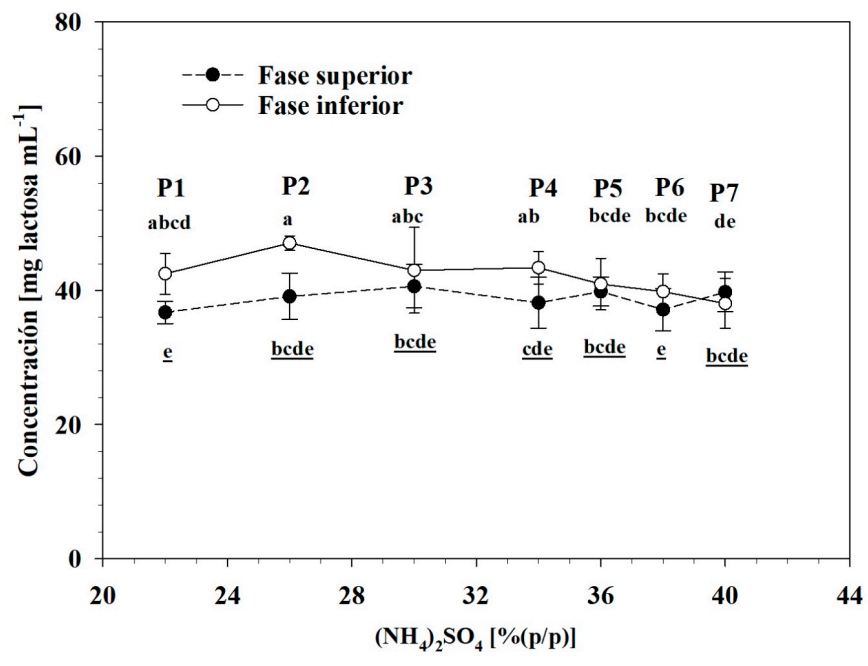

Figura 7. Concentración de lactosa en la fase superior e inferior de los sistemas evaluados (letras subrayadas indican fase inferior correspondiente). Letras diferentes indican diferencias significativas, de acuerdo a la prueba de Tukey $(p<0.05)$.

Figure 7. Lactose concentration at top and bottom phases from evaluated operation points (underlined words indicate corresponding bottom phase). Different letters indicate significant differences, according to Tukey's test ( $p$ $<0.05)$.

nio y encontraron que el valor de este parámetro disminuyó a medida que la concentración del polímero también disminuyó, lo que indicó que en ese sistema la lactosa migró de la fase superior a la fase inferior de los sistemas. Suarez Ruiz et al. (2020) señalaron que azúcares como la glucosa migran hacia la fase más hidratada o con más agua disponible (frecuentemente la fase inferior) en un sistema polímero/sal. Al respecto, al demandar menor agua disponible que el PEG, la proteína no indujo un efecto de exclusión sobre la lactosa similar al de este polímero y, por ello, la lactosa se repartió entre ambas fases. Sin embargo, el coeficiente de partición se afecta por el volumen de las fases y se requiere una valoración de las cantidades absolutas que se obtuvieron en cada fase con base en los rendimientos de separación.

\section{Rendimientos de separación}

El rendimiento de separación de proteína en la fase superior $\left(Y_{\text {pro }}^{\text {top }}\right.$ ) varió de 31.77 a 83.82 \% con la transición entre los estados P1 y P7 y con un comportamiento sigmoidal (Figura 8). A medida que se incrementó la concentración de $\left(\mathrm{NH}_{4}\right)_{2} \mathrm{SO}_{4}$ se incrementó el efecto de exclusión salina de este compuesto sobre la proteína (Gomes et al., 2017), con lo cual mayores cantidades de proteína de lactosuero se separaron en la fase superior. Este efecto de exclusión salina fue moderado en la transición de los estados P1 a P2, lo que indicó que con concentraciones de $\left(\mathrm{NH}_{4}\right)_{2} \mathrm{SO}_{4}$ entre 22 y 26 $\%$ la solubilidad de las proteínas no se vio significativamente afectada, pero entre 26 y $34 \%$ el efecto de exclusión salina se incrementó de forma muy importante y, para concentraciones mayores de la sal, el incremento en la separación de estos compuestos en la fase superior fue moderado.

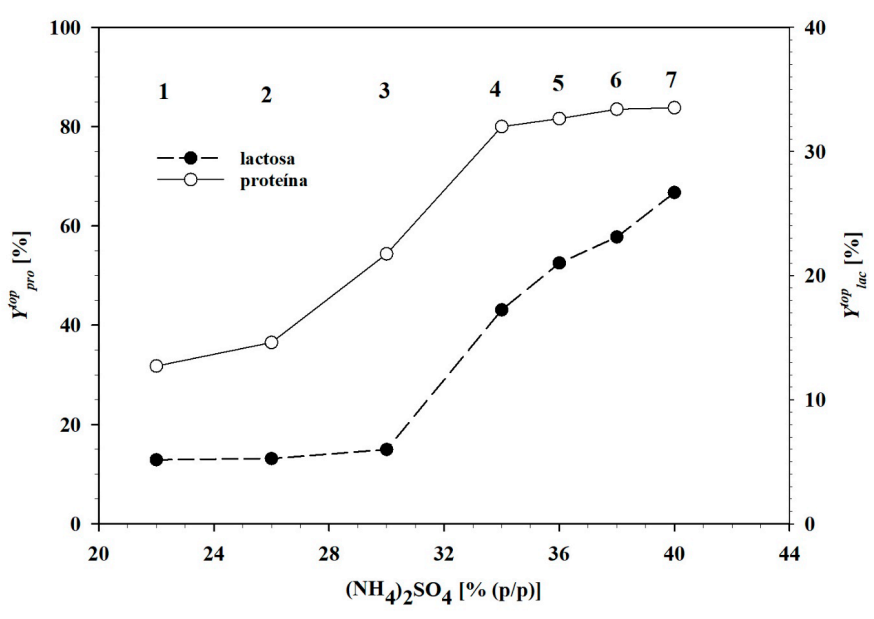

Figura 8. Rendimiento para la fase superior de los sistemas experimentales evaluados. a) relación entre el rendimiento en la fase superior para lactosa y proteína con el porcentaje de volumen. b) Relación entre la concentración de sulfato de amonio con el rendimiento de lactosa y proteína en la fase superior.

Figure 8. Yield of top and bottom phase of evaluated operation points. a) Relation between top phase lactose and protein yield, and volume percentage. b) Top phase relation between ammonium sulphate concentration, and lactose and protein yield.

El rendimiento de separación de lactosa en la fase superior de los sistemas $\left(Y_{l a c}^{t o p}\right)$ presentó valores entre 5.16 y $5.98 \%$ en los sistemas $\mathrm{P} 1$ a $\mathrm{P} 3$, pero registró un incremento en la transición de P3 a P7 hasta valores cercanos a $29 \%$ (Figura 8), lo que indicó que la recuperación de lactosa en la fase inferior de los sistemas P1 a P3 varió entre 94.02 y $94.84 \%$ y se redujo hasta valores cercanos a $71 \%$ con la transición de P3 a P7. En tal sentido, aunque el coeficiente de partición tuvo valores cercanos a la unidad, el rendimiento de separación indicó que las mayores cantidades absolutas de lactosa permanecieron en la fase inferior, en forma también congruente a lo reportado por Domínguez-Puerto et al. (2018).

Con base en un análisis conjunto de separación de proteína en la fase superior y de lactosa en la fase inferior, se encontró que los sistemas P3 y P4 tuvieron el mejor potencial para atender la separación de estos compuestos. El sistema P3 se formó con $30 \%$ de $\left(\mathrm{NH}_{4}\right)_{2} \mathrm{SO}_{4}$ y permitió que el $54.4 \%$ proteína y el $5.98 \%$ de lactosa del lactosuero se separaron en la fase superior, en tanto que con el sistema P4, formado por $34 \%$ de $\left(\mathrm{NH}_{4}\right)_{2} \mathrm{SO}_{4}$, el $80.01 \%$ de proteína y $17.22 \%$ de lactosa se separaron en la misma fase. Sin embargo, en virtud de que el principal objetivo es recuperar la proteína del lactosuero, se aceptó que el mejor sistema para cumplir este propósito fue el que contuvo $34 \%$ de sulfato de amonio (P4), lo cual coincidió con el reporte de Domínguez-Puerto et al. (2018). Con esta base, el uso de la técnica de extracción acuosa en dos fases tiene potencial para recuperar proteínas del lactosuero y el uso posterior de éstas en estrategias de fortificación de alimentos. 


\section{CONCLUSIONES}

El sistema $\left(\mathrm{NH}_{4}\right)_{2} \mathrm{SO}_{4}$ /proteína puede formar sistemas bifásicos acuosos con concentraciones de sulfato de amonio mayores a 22 \%. La separación mostró sistemas donde el volumen de la fase superior fue significativamente mayor al de la fase inferior, pero el incremento de la concentración de sal causó que el volumen de la fase superior aumentara con relación al de la inferior. La proteína de lactosuero se concentró en mayor cantidad en la fase superior que en la inferior y por ello, la estrategia de mezclar lactosuero con sulfato de amonio constituyó una alternativa viable para recuperar las proteínas en porcentajes mayores al 80 \%. Aunque la lactosa mostró concentraciones similares en ambas fases, el desarrollo de sistemas con volumen en fase superior menor al de la fase inferior permitió que aproximadamente $79 \%$ de este disacárido fuera separado en la fase inferior. La técnica de extracción acuosa en dos fases tiene potencial para separar proteínas del lactosuero con objeto de que éstas se utilicen en estrategias de fortificación de alimentos.

\section{AGRADECIMIENTOS}

Los autores agradecen a la Unidad Tecnológica Lechera (UTL) de la Universidad Autónoma Chapingo por las facilidades para el suministro de lactosuero para la realización de esta investigación.

\section{REFERENCIAS}

Alcântara, L.A.P., Minim, L.A., Minim, V.P.R., Bonomo, R.C.F., da Silva, L.H.M., y da Silva, M.D.C.H. 2011. Application of the response surface methodology for optimization of whey protein partitioning in PEG/phosphate aqueous two-phase system. Journal of Chromatography B, 879, 1881-1885. DOI:10.1016/j.jchromb.2011.05.007

Balasubramaniam, D., Wilkinson, C., Cott, K. V., Zhang, C. 2003. Tobacco protein separation by aqueous two-phase extraction. Journal of Chromatography A, 989, 119-129.

Bensch, M., Selbach, B. y Hubbuch, J. 2007. High throughput screening techniques in downstream processing: Preparation, characterization and optimization of aqueous two-phase systems. Chemical Engineering Science, 62, 2011-2021.

Capezio, L., Romanini, D., Picó, G. A. y Nerli, B. 2005. Partition of whey milk proteins in aqueous two-phase systems of polyethylene glycol-phosphate as a starting point to isolate proteins expressed in transgenic milk. Journal of Chromatography B, 819, 25-31.

da Rocha, P. P., Barbosa, M. A., Rodrigues, D. L. L., Moreira, M. C. R., y Mendes, S. L. H. Hespanhol, D. S. M. C. 2011. Fluid Phase Equilibria Phase diagram and thermodynamic modeling of PEO + organic salts $+\mathrm{H}_{2} \mathrm{O}$ and PPO + organic salts $+\mathrm{H}_{2} \mathrm{O}$ aqueous two-phase systems. Fluid Phase Equilibria, 305, 1-8. https://doi.org/10.1016/j.fluid.2011.02.013

Domínguez-Puerto, R., Valle-Guadarrama, S., Guerra-Ramírez, D. y Hahn-Schlam, F. 2018. Purification and concentration of cheese whey proteins through aqueous two phase extraction. CyTA - Journal of Food, 16, 452-459.

Dubois, M., Gilles, K. A., Hamilton, J. K., Rebers, P. A. y Smith, F. 1956. Colorimetric Method for Determination of Sugars and Related Substances. Analytical Chemistry, 28, 350-356.
Duong-Ly, K. C. y Gabelli, S. B. 2014. Chapter Seven - Salting out of Proteins Using Ammonium Sulfate Precipitation. In: LORSCH, J. (ed.) Methods in Enzymology. Academic Press.

Gomes, J., Ferreira, S., Reinert, O., Gandol, R., Ayra, L., Santos, V., et al. 2017. Fluid phase equilibria evaluation of saltingout effect in the liquid e liquid equilibrium of aqueous two-phase systems composed of 2-propanol and $\mathrm{Na}_{2} \mathrm{SO}_{4} /$ $\mathrm{MgSO}_{4}$ at different temperatures. Fluid Phase Equilibria, 450, 184-193. https://doi.org/10.1016/j.fluid.2017.08.001

Jara, F. y Pilosof, A. M. R. 2011. Partitioning of a-lactalbumin and $\beta$-lactoglobulin in whey protein concentrate/ hydroxypropylmethylcellulose aqueous two-phase systems. Food Hydrocolloids, 25, 374-380.

Jelen, P., y Tossavainen, O. 2003. Low lactose and lactose-free milk and dairy products - prospects, technologies and applications. Australian Journal of Dairy Technology, 58, 161-165.

Jiang, Bin; Zhang, Xiaoqing; Yuan, Yongqiang; Qu, Yuxiao; Feng, Zhibiao. 2017. Separation of antioxidant peptides from pepsin hydrolysate of whey protein isolate by ATPS of EOPO co-polymer (UCON)/phosphate. Scientific Reports, 7 (1), 13320, 1-12. DOI: 10.1038/s41598-017-13507-9

Kalaivani, S., y Regupathi, I. 2015. Synergistic extraction of a-lactalbumin and $\beta$-lactoglobulin from acid whey using aqueous biphasic system: process evaluation and optimization. Separation and Purification Technology, 146, 301-310. doi:10.1016/j.seppur.2015.03.057

Kamizake, N. K. K., Gonçalves, M. M., Zaia, C. T. B. V. y Zaia, D. A. M. 2003. Determination of total proteins in cow milk powder samples: a comparative study between the Kjeldahl method and spectrophotometric methods. Journal of Food Composition and Analysis, 16, 507-516.

Lobato-Calleros, C., Lozano-Castañeda, I. y Vernon-Carter, E. J. 2009. Textura y microestructura de quesos tipo panela bajos en grasa y en colesterol: diferentes metodologías. Ingeniería Agrícola y Biosistemas, 1.

Madureira, A. R., Pereira, C. I., Gomes, A. M. P., Pintado, M. E. y Xavier Malcata, F. 2007. Bovine whey proteins - Overview on their main biological properties. Food Research International, 40, 1197-1211.

Merchuk, J. C., Andrews, B. A. y Asenjo, J. A. 1998. Aqueous two-phase systems for protein separation: Studies on phase inversion. Journal of Chromatography B: Biomedical Sciences and Applications, 711, 285-293.

Mokhtarani, B., Mortaheb, H. R., Mafi, M., y Amini, M. H. 2011. Partitioning of a-lactalbumin and $\beta$-lactoglobulin in aqueous two-phase systems of polyvinylpyrrolidone and potassium phosphate. Journal of chromatography. B, Analytical technologies in the biomedical and life sciences, 879(11-12), 721-726. DOI: 10.1016/j.jchromb.2011.02.007

Murillo-Martínez, M. M., Tello-Solís, S. R., García-Sánchez, M. A., y Ponce-Alquicira, E. 2013. antimicrobial activity and hydrophobicity of edible whey protein isolate films formulated with nisin and/or glucose oxidase. Journal of Food Science, 78(4), M560-M566. https://doi. org/10.1111/1750-3841.12078

Nandini, K. E., y Rastogi, N. K. 2011. Integrated Downstream Processing of Lactoperoxidase from Milk Whey Involving Aqueous Two-Phase Extraction and Ultrasound-Assisted Ultrafiltration. Applied Biochemistry and Biotechnology, 163(1), 173-185. DOI: 10.1007/s12010-010-9026-9 
Patel, S. 2015. Emerging trends in nutraceutical applications of whey protein and its derivatives. Journal of Food Science and Technology, 52(11), 6847-6858. https://doi.org/10.1007/ s13197-015-1894-0

Pereira Alcântara, L. A., Vieira Amaral, I., Ferreira Bonomo, R. C., Mendes da Silva, L. H., Hespanhol da Silva, M. d. C., Rodrigues Minim, V. P., y Minim, L. A. 2014. Partitioning of a-lactalbumin and $\beta$-lactoglobulin from cheese whey in aqueous twophase systems containing poly (ethylene glycol) and sodium polyacrylate. Food and Bioproducts Processing, 92, 409-415. https://doi.org/10.1016/j.fbp.2013.09.006

Perumalsamy, M., y Murugesan, T. (2012). Extraction of cheese whey proteins (a-lactalbumin and $\beta$-lactoglobulin) from dairy effluents using environmentally benign aqueous biphasic system. International Journal of Chemical and Environmental Engineering, 3(1), 50-54.

Prazeres, A. R., Carvalho, F. y Rivas, J. 2012. Cheese whey management: A review. Journal of Environmental Management, 110, 48-68.

Raja, S., Murty, V. R., Thivaharan, V., Rajasekar, V. y Ramesh, V. 2011. Aqueous two phase systems for the recovery of biomolecules-a review. Science and Technology, 1, 7-16.

Rodríguez-Salazar, N., y Valle-Guadarrama, S. 2020. Separation of phenolic compounds from roselle (Hibiscus sabdariffa) calyces with aqueous two-phase extraction based on sodium citrate and polyethylene glycol or acetone. Separation Science and Technology (Philadelphia), 55(13), 2313-2324. https://doi.org/10.1080/01496395.2019.1634730

Rosa, P. A. J., Ferreira, I. F., Azevedo, A. M., y Aires-Barros, M. R. 2010. Aqueous two-phase systems: A viable platform in the manufacturing of biopharmaceuticals. Journal of Chromatography A, 1217(16), 2296-2305. https://doi. org/10.1016/j.chroma.2009.11.034

Sadeghi, F., Kadkhodaee, R., Emadzadeh, B., y Nishinari, K. 2021. Effect of sucrose on phase and flow behavior of protein-polysaccharide mixtures. Food Hydrocolloids, 113(November 2020), 106455. https://doi.org/10.1016/j. foodhyd.2020.106455
Sadeghi, R., y Maali, M. 2016. Toward an understanding of aqueous biphasic formation in polymer-polymer aqueous systems. Polymer, 83, 1-11. https://doi.org/10.1016/j. polymer.2015.11.032

SAS Institute, Inc. 1999. SAS/STAT ${ }^{\oplus}$ User's Guide, Version 8. SAS Institute Inc., Cary, NC, USA. 3884 p.

Spyropoulos, F., Portsch, A. y Norton, I. T. 2010. Effect of sucrose on the phase and flow behaviour of polysaccharide/protein aqueous two-phase systems. Food Hydrocolloids, 24, 217-226.

Suarez Ruiz, C. A., Baca, S. Z., Van Den Broek, L. A. M., Van Den Berg, C., Wijffels, R. H. y Eppink, M. H. M. 2020. Selective fractionation of free glucose and starch from microalgae using aqueous two-phase systems. Algal Research, 46, 101801.

The Mathworks, Inc. 1990-2008. Optimization Toolbox ${ }^{\text {TM }} 4$ User's Guide. Natick, Massachusetts, USA: The Mathworks Inc. 575 p.

Turecek, P. L., Bossard, M. J., Schoetens, F. y Ivens, I. A. 2016. PEGylation of Biopharmaceuticals: A Review of Chemistry and Nonclinical Safety Information of Approved Drugs. Journal of Pharmaceutical Sciences, 105, 460-475.

Wingfield, P. T. 2016. Protein Precipitation Using Ammonium Sulfate. Current Protocols in Protein Science, 84, A.3F.1A.3F.9.

Yadav, J. S. S., Yan, S., Pilli, S., Kumar, L., Tyagi, R. D. y Surampalli, R. Y. 2015. Cheese whey: A potential resource to transform into bioprotein, functional/nutritional proteins and bioactive peptides. Biotechnology Advances, 33, 756-774.

Yang, Z., Liu, X.-J., Chen, C. y Halling, P. J. 2010. Hofmeister effects on activity and stability of alkaline phosphatase. Biochimica et Biophysica Acta (BBA) - Proteins and Proteomics, 1804, 821-828.

Zhang, H., Jiang, B., Feng, Z.-B., Qu, Y.-X., y Li, X. 2016. Separation of $a$-Lactalbumin and $\beta$-Lactoglobulin in Whey Protein Isolate by Aqueous Two-phase System of Polymer/ Phosphate. Chinese Journal of Analytical Chemistry, 44(5), 754-759. DOI: 10.1016/S1872-2040(16)60932-5 